\title{
MATHEMATICAL MODELS CONSTRUCTION FOR BUILDING ROBOTS WITH DUE ACCOUNT OF ELASTIC DEFORMATIONS OF MECHANISMS
}

\author{
Alexej Bulgakov** \\ Thomas Bock* \\ * Technical University Munich, Germany, thomas.bock@bri.ar.tum.de \\ ** South Russian State Technical University (NPI), a.bulgakow@gmx.de
}

\begin{abstract}
The paper presents the formulation of general approach to construction of structural models for elastic manipulators, which can be presented in the form of collection of connected in series bodies experiencing elastic deformation, and suggests the methods of changing over to such structure. The paper describes in detail the method of constructing mathematical models for manipulators with elastic couplings, which is based on the principle of finite partitions and differs by introducing fictitious degrees of freedom in the points critical to deformations. It also considers an example of constructing model for a building-mounting robot on the basis of the suggested method.
\end{abstract}

KEYWORDS: building robots, mathematical models, elastic deformations.

\section{INTRODUCTION}

One of the directions of civil engineering intensification, improvement of builders' working conditions is designing and introduction of robots for civil engineering work and also development on their basis of robotic systems. A specific feature of building robots is considerable dimensions of manipulators' links, action of considerable forces and moments, causing elastic deformations of links. As a result, building robots control should be carried out with due account of elastic deformations of a manipulating mechanism. Therefore, the given paper considers the problems of constructing mathematical models for building robots taking into account elastic deformations in a manipulating system, which appear under the influence of dynamic, and wind loads.

\section{GENERAL CONCEPT OF CONSTRUCTING MODELS FOR ELASTIC MANIPULATORS}

Let us formulate general concept of constructing elastic manipulators structure for building robots. According to the principle of finite partitions such manipulators can be regarded as a collection of rigid bodies with elastic coupling in series. In terms of the above mentioned it is suggested to consider elastic manipulator models while constructing them in the form of the structure, which in addition to actual degrees of freedom contains fictitious ones imitating elastic deformations of links (fig.1).

The change-over to this structure is performed on the basis of the manipulating system analysis so as to define points for

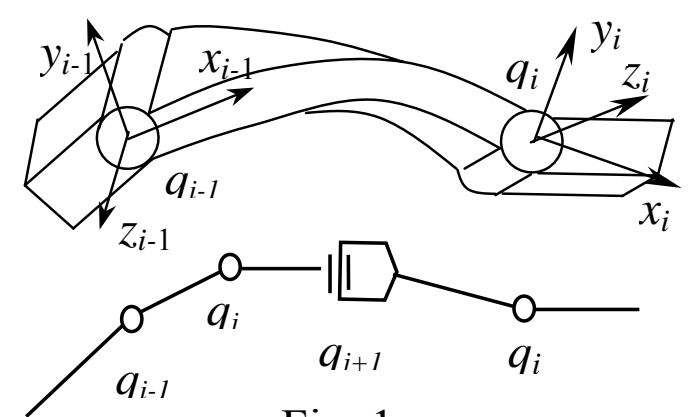

Fia. 1

deformed links which are most critical to deformations (PCD). In the capacity of such points those are chosen where the ratio of ultimate stress during manipulator operation to unit strain of the structure element reaches maximal value.

The structure element incorporating PCD is divided in this point into two bodies connected by a fictitious hinge (fig.1). When constructing mathematical model the hinge is replaced by three mutually perpendicular rotational degree of freedom 
where the center of rotation coincides with PCD.

Although, depending on the kind of links deformation the number of degrees of freedom of a fictitious hinge can be reduced to one. Introduction of fictitious degrees of freedom makes it possible to take accounts of shifts stipulated by availability of elastic deformations. When constructing a dynamic model for the manipulator of such structure we introduce an equation for generalized forces of fictitious links so as to take into account elastic forces. Parameters of their equations are identified from the condition of the best correspondence of a mathematical model to a real manipulator. If we fail to obtain the required accuracy of the model parameters, then we try to find one more PCD with subsequent introduction into the model fictitious degrees of freedom.

\section{MATHEMATICAL MODELS FOR BUILDING ROBOTS}

For mathematical description of the suggested manipulator structure we use a matrix method. In accordance with matrix of the robot coordinate system transformation $\quad T_{c}\left(\mathbf{q}_{c}\right)=T_{b c} A_{0}\left(\mathbf{q}_{c}\right)$ Cartesian coordinates of the manipulator end-effector are equal to :

$$
\overline{\mathbf{x}}(\mathbf{q})=\mathbf{f}(\mathbf{q}, \mathbf{d x})=\left\{(T(\mathbf{q}) \boldsymbol{\Delta} \mathbf{x})_{j}\right\}_{j}
$$

where $\Delta \mathbf{x}$ is tool or gripper coordinates in the system of coordinates of the manipulator outlet link. The end-effector orientation can be determined by comparing submatrix elements of the rotation matrix $T_{c}(\mathbf{q})$ and the matrix of Euler angles rotation, as a result we obtain:

$$
\varphi=\left(\begin{array}{c}
\operatorname{arctg} 2\left(-T^{(1,2)}, T^{(2,2)}\right) \\
\arcsin T^{(3,2)} \\
\operatorname{arctg} 2\left(-T^{(3,1)}, T^{(3,3)}\right)
\end{array}\right)
$$

where $\operatorname{arctg} 2(\cdot)$ is the function reverse to tangent.

For convenience of the manipulator models analysis we divide the generalized coordinates into controlled $\mathbf{q}^{\prime}=\left(q_{1}, q_{2} \ldots q_{m}\right)^{\mathrm{T}} \quad$ and $\quad$ generalized coordinates describing deformation of the manipulator

elements, $\mathbf{q}^{\prime \prime}=\left(q_{1}, q_{2} \ldots q_{m-1}\right)^{\mathrm{T}}$ at the same time $\mathbf{q}=\mathbf{q}^{\prime} \cup \mathbf{q}^{\prime \prime}$. When solving reverse task about position and speed relative to controlled coordinates $q^{\prime}$ we consider that fictitious generalized coordinates $q^{\prime \prime}$ and their speeds of are known (measured or calculated for the model). Analytical solution of the non-linear trigonometric equation (1) for the variables $q^{\prime}$ is difficult because of availability of non-linearities. For building robots control it is convenient to perform solution of inverse kinematic problem in terms of iterative approximation and algorithm for numerical solution of a non-linear trigonometric equation. Therefore, having transformed equations (1) and using expansion into a Maklaren series for $q_{1}^{\prime}, \quad q_{2}^{\prime} \ldots q_{n}^{\prime}$ in the points $\quad q_{1}^{\prime}=q_{1}^{\prime(i)}, \quad q_{2}^{\prime(i)} \ldots q_{n}^{\prime}=q_{n}^{\prime(i)} \quad$ we obtain:

$$
\Delta q_{i}^{(k)}=-\sum_{j=1}^{3} \xi_{j}\left(q_{1}^{(i)}\right) /\left.\frac{d \xi_{j}\left(q_{1}\right)}{d q_{1}}\right|_{q_{1}=q_{1}^{(i)}},
$$

where $i=1,2 \ldots n$ is a number of iterative approximation.

Going over between iterations is performed by the formula

$$
q_{1}^{\prime(k+1)}=q_{1}^{(k)}+\Delta q_{1}^{(k)}
$$

The iterations begin from the values of $q_{i}^{\prime}=q_{i}^{(0)}$, where $q_{i}^{\prime(0)}$ is the current position and they continue until the condition of: $q_{i}^{\prime}-q_{i}^{\prime(k)}<\varepsilon_{i}$ is fulfilled, where $\varepsilon_{i}$ is accuracy of positioning.

Solution of direct kinematic problem for speed and acceleration will be obtained by differentiating equation (1):

$$
\begin{aligned}
& \&=G(\mathbf{q}, \Delta \mathbf{x}) \cdot \&, \\
& =G(\mathbf{q}, \Delta \mathbf{x}) \cdot \&+q(\mathbf{q}, \&, \Delta \mathbf{x}) \cdot \&,
\end{aligned}
$$

where $G(\mathbf{q}, \Delta \mathbf{x})$ is a Jacobean matrix [3*n] equal to

$$
G(\mathbf{q}, \Delta \mathbf{x})=\left\{U_{n i}^{(j n)}(\mathbf{q}) \cdot \Delta \mathbf{x}\right\}_{j, i},
$$




$$
\begin{aligned}
& \mathcal{G}(\mathbf{q}, \boldsymbol{\phi}, \Delta \mathbf{x})=\left\{\left(\sum_{k=1}^{i} U_{n i k}(\mathbf{q}) \Delta \mathbf{x} \cdot \&_{k}\right)_{j}\right\}_{j, i}, \\
& i=\overline{1, n} j=\overline{1,3} \text {. }
\end{aligned}
$$

Having performed partition of the generalized coordinates into controlled and uncontrolled ones we obtain:

$$
\begin{aligned}
& \mathbf{x}=G^{\prime}(\mathbf{q}, \Delta \mathbf{x}) \mathcal{q}^{\&}+G^{\prime \prime}(\mathbf{q}, \boldsymbol{\Delta x}) \mathcal{\alpha}^{\prime \prime},
\end{aligned}
$$

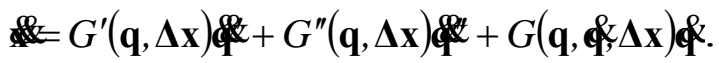

The generalized speeds and accelerations of the controlled coordinates are calculated from expressions:

$$
\begin{aligned}
& \mathcal{q}^{\prime}=G^{\prime}(\mathbf{q}, \Delta \mathbf{x})^{-1}\left(\mathbb{\&}-G^{\prime \prime}(\mathbf{q}, \Delta \mathbf{x}) \cdot \mathbf{q}^{\prime \prime}\right), \\
& G^{\prime}(\mathbf{q}, \Delta \mathbf{x})^{-1} \text {. } \\
& \text {. } \left.\left(G^{\prime \prime}(\mathbf{q}, \mathbf{\Delta x}) \mathbf{q}^{\prime \prime}-G(\mathbf{q}, \& \mathbf{\Delta} \mathbf{x})\right)^{q}\right) \text {. }
\end{aligned}
$$

The robot dynamics with due account of forces arising in elastic elements will be described by the dependency:

$$
\mathbf{M}=D(\mathbf{q}) \mathbf{b}(\mathbf{q}, \boldsymbol{\phi})-\sum_{k=1}^{l} G^{\mathrm{T}}\left(\mathbf{q}, \Delta \mathbf{x}^{(k)}\right) \mathbf{F}_{s}^{(k)}-\mathbf{M}_{w},
$$

whence

$$
\begin{aligned}
& D^{-1}(\mathbf{q}) \cdot\left(\mathbf{M}-\mathbf{b}(\mathbf{q}, \mathbf{q})+\mathbf{M}_{w}+\right. \\
& \left.+\sum_{k=1}^{l} G^{\mathrm{T}}\left(\boldsymbol{\Delta} \mathbf{x}^{(k)}, \mathbf{q}\right) \mathbf{F}_{s}^{(k)}\right),
\end{aligned}
$$

where $\mathbf{M}$ is the vector [n*1] of generalized forces arising in the manipulator joints; $D(\mathbf{q})$ is the dynamics matrix [n*n] elements of which are equal; $\mathbf{b}(\mathbf{q}, \boldsymbol{q})$ is the vector [ $\mathrm{n} * 1]$ of Carioles, centrifugal and gravitational forces; $\mathbf{F}_{s}^{(k)}$ is the vector of forces [ $3 * 1]$ arising in $k$-th point of an endeffector interaction with surface; $\Delta \mathbf{x}^{(k)}$ is the shift vector [3*1] of the point of application of $k$-th external force; $\mathbf{M}_{w}$ is the vector $[\mathrm{n} * 1]$ of generalized forces arising in elastic elements of the manipulator links.

Using the operator of the robot generalized coordinates $\mathbf{q}$ division into the controlled ones $\mathbf{q}^{\prime}$ and the introduced ones for imitating elastic deformation $\mathbf{q}^{\prime \prime}$ we transform equation (10) to the form:

$$
\begin{aligned}
\mathbf{M}^{\prime}=D^{\prime}(\mathbf{q}) & +D(\mathbf{q}) \mathbf{q}^{\prime}+\mathbf{b}^{\prime}(\mathbf{q}, \mathbf{\phi})- \\
& -\sum_{k=1}^{3} G^{\prime \mathrm{T}}\left(\mathbf{d} \mathbf{x}^{(k)}, \mathbf{q}\right) \mathbf{F}_{s}^{(k)}-\mathbf{M}_{w}^{\prime},
\end{aligned}
$$

$$
\begin{aligned}
\mathbf{M}^{\prime \prime}=\grave{D}^{\mathrm{T}} & (\mathbf{q}) \mathbf{q}^{\prime \prime}(\mathbf{q}) \mathbf{q}^{\mathbf{q}}+\mathbf{b}^{\prime \prime}(\mathbf{q}, \boldsymbol{\phi})- \\
& -\sum_{k=1}^{3} G^{\prime \prime}\left(\mathbf{d} \mathbf{x}^{(k)}, \mathbf{q}\right) \mathbf{F}_{s}^{(k)}-\mathbf{M}_{w}^{\prime \prime} .
\end{aligned}
$$

The obtained model of elastically deforming manipulating system of a robot in the form of matrix is convenient for constructing a building robot model and for constructing its control algorithms.

\section{MODEL OF A BUILDING- MOUNTING ROBOT}

Using the described methods for manipulating systems modeling we construct kinematic and dynamic models of the mounting robot manipulator. Having analysed the suggested design of the manipulator we define the number of degrees of freedom including fictitious ones (fig.2). The characteristic feature of the manipulator is the possibility of appearing elastic deformations of the last link as the most subject to deformations because of great ratio of length to section area. In accordance with the presented methods we define PCD on this link and introduce into it a hinge with two mutually perpendicular rotating couples rotating axes of which are perpendicular to the

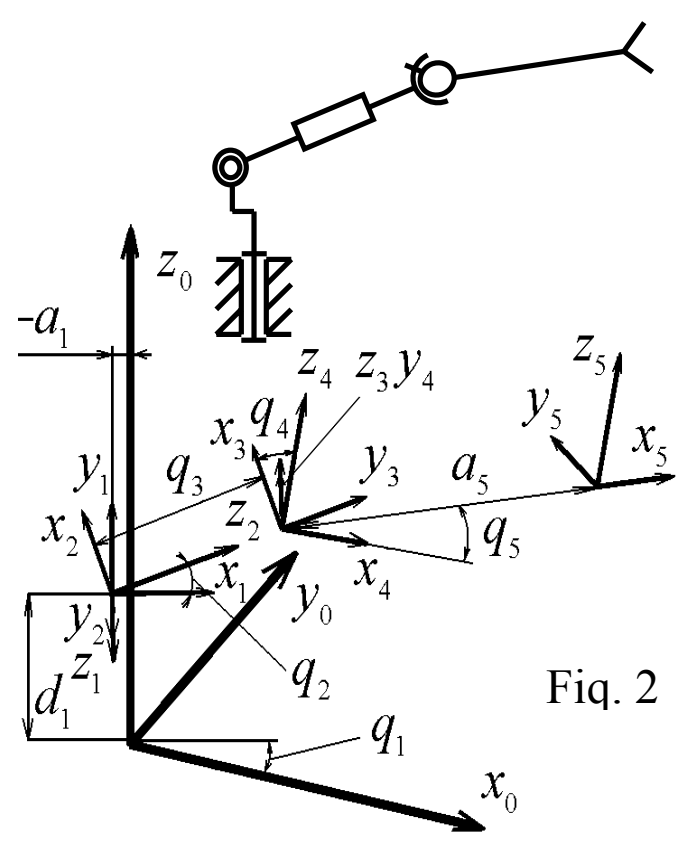

longitudinal axis.

According to fig. 2 we put down the transition probability matrix from the absolute system of coordinates connected 
with place of the robot installation to the gripper system of coordinates:

$T_{r}\left(\mathbf{q}_{r}\right)=T_{b r}\left(\varphi_{b r}, \mathrm{x}_{b r}\right) \cdot R\left(a_{r 1}, d_{r 1}, \pi / 2, q_{r 1}\right)$.

$\cdot R\left(0,0, \pi / 2, \pi / 2+q_{r 2}\right) \cdot R\left(0, q_{r 3}, 0,0\right)$

Then the direct task of kinematics with respect to the point of the gripper attachment will be described by the system of equations in the form of

$$
\begin{aligned}
& \mathbf{x}_{r}\left(\mathbf{q}_{r}\right)=\mathbf{f}_{r}\left(\mathbf{q}_{r}, \mathbf{z}\right)= \\
& =\left(\begin{array}{c}
\left(a_{r 1}+q_{r 3} \cos q_{r 2}\right) \cos \left(q_{r 1}+\varphi_{b r}\right)+x_{b r 1} \\
\left(a_{r 1}+q_{r 3} \cos q_{r 2}\right) \sin \left(q_{r 1}+\varphi_{b r}\right)+x_{b r 2} \\
d_{r 1}+q_{r 3} \sin q_{r 2}+x_{b r 3}
\end{array}\right) .
\end{aligned}
$$

In order to take into account deformations of the last link we introduce additional systems of coordinates (fig.3) in the point of critical deformation located in $a_{5}$ distance relative to the center of the 2-nd degree of freedom. For convenience of the generalized coordinates count we assume

$\theta_{r 2}=\pi / 2+q_{r 2}, \quad \theta_{r 4}=90+q_{r 4}, q_{r 4}=0$, $q_{r 5}=0, q_{\text {ж, }, 33}=q_{\text {д, }, r 3}+a_{\text {д, }, 5}$.

In terms of the suggested scheme the direct task of kinematics for the manipulator with deformed link can be described by the equation

$$
\mathbf{x}_{r}\left(\mathbf{q}_{r}\right)=\left(\begin{array}{c}
\lambda C_{q}-a_{r 5} S_{q} \sin q_{r 5}+x_{b r 1} \\
\lambda S_{q}+a_{r 5} C_{q} \sin q_{r 5}+x_{b r 2} \\
a_{r 5} S_{q q} \cos q_{r 5}+\rho_{1} \sin q_{r 2}+\rho_{2}
\end{array}\right)
$$

where $\rho_{1}=q_{r 3}+d_{r 3}, \rho_{2}=d_{r 1}+x_{b r 3}$,

$$
\begin{aligned}
C_{q} & =\cos \left(q_{r 1}+\varphi_{b r}\right), S_{q}=\sin \left(q_{r 1}+\varphi_{b r}\right), \\
\mathrm{S}_{q q} & =\sin \left(q_{r 2}-q_{r 4}\right), \lambda=\left(q_{r 3}+d_{r 3}\right) \cos q_{r 2}+ \\
& +a_{r 5} \cos \left(q_{r 2}-q_{r 4}\right) \cos q_{r 5}+a_{r 1} .
\end{aligned}
$$

Let us introduce vector division of the robot generalized coordinates $q_{r}$ into the controlled ones $q_{r}^{\prime}=\left(q_{r 1}, q_{r 2}, q_{r 3}\right)^{\mathrm{T}}$ and the fictitious ones $q_{r}^{\prime \prime}=\left(q_{r 4}, q_{r 5}\right)^{\mathrm{T}}$.

On the basis of the suggested kinematic model we build a dynamic model for the robot. The manipulator dynamics equation for the $k$-th arm of the robot will be written in the following form

$$
\begin{aligned}
& \mathbf{M}_{r}^{\prime}=D_{r}^{\prime}{ }_{r}+D_{r}+\mathbf{b}_{r}^{\prime}+\left(G_{r}^{\prime}\right)^{\mathrm{T}}\left(\mathbf{d} \mathbf{x}_{r}, \mathbf{q}_{r}\right) \mathbf{F}_{s}, \\
& \mathbf{M}_{r}^{\prime \prime}=D_{r}^{\mathrm{T}}+D_{r}^{\prime \prime} \mathbf{q}_{r}+\mathbf{b}_{r}^{\prime \prime}+\left(G_{r}^{\prime \prime}\right)^{\mathrm{T}}\left(\mathbf{d} \mathbf{x}_{r}, \mathbf{q}_{r}\right) \mathbf{F}_{r} .
\end{aligned}
$$

where $\mathbf{d x _ { r }}$ is the shift of the robot attachment point with the installed element in the system of coordinates of the last link.

The generalized moment of the controlled degrees of freedom $\mathbf{M}_{r}^{\prime}$ is created by electromagnet forces of the robot drives and the generalized forces in the fictitiously introduced degrees of freedom are equal to

$$
\mathbf{M}_{r}^{\prime \prime}=-\alpha_{r} \mathbf{q}_{r}^{\prime \prime}-\beta_{r} \phi_{r}^{\prime \prime}
$$

where $\alpha_{r}, \beta_{r}$ are diagonal matrices of toughness and dissipation of the robot last arm relative to the point critical to deformation.

\section{CONCLUSION}

The described methods of mathematical models construction for building robots makes it possible to eliminate difficulties connected with elastic deformations in a mechanical system and also with the availability of branching and closed structures. Application of fictitious degrees of freedom for the elastic forces imitation allows to simplify mathematical models of building robots to a great extent. The model equations being obtained during calculations can be used for developing control algorithms of building robots. The truth of the assumptions adopted during the development of mathematical models was verified by physical modeling and computer simulation. 